\title{
Optical Modelling using Gaussian Beam Modes for the Terahertz Band
}

\author{
Créidhe O'Sullivan ${ }^{* a}$, John A. Murphy ${ }^{\mathrm{a}}$, Marcin L. Gradziel ${ }^{\mathrm{a}}$, John Lavelle ${ }^{\mathrm{a}}$, Tully Peacocke, \\ Neil Trappe ${ }^{a}$, Gareth S. Curran ${ }^{b}$, David R. White ${ }^{c}$, Stafford Withington ${ }^{d}$ \\ ${ }^{a}$ Department of Experimental Physics, National University of Ireland, Maynooth, \\ Maynooth, Co. Kildare, Ireland; \\ bInstitute of Technology Blanchardstown, Blanchardstown Rd. North, Dublin 15, Ireland; \\ ${ }^{\mathrm{c}}$ Department of Computing, Institute of Technology Tallaght, Dublin 24, Ireland; \\ ${ }^{\mathrm{d}}$ Cavendish Laboratory, University of Cambridge, Madingley Road, CB3 OHE, United Kingdom
}

\begin{abstract}
Special approaches unique to the waveband are required for the modelling of terahertz optical systems. Ray tracing is inadequate because in typical instruments the propagating beams are not very many wavelengths in diameter and a "quasi-optical" approach is required in which Fresnel diffraction effects can be efficiently and accurately simulated. Typically, it is also necessary to be able to simulate the coupling of quasi-optical beams to feed antenna structures to predict optical performance. In many systems the beams can be considered to be coherent and their propagation through a beam guide consisting of re-focussing elements can be efficiently modelled using modal analysis, especially useful for quick design purposes, beam control and optimisation. This modal approach has been extended to allow for aberrations and truncation particularly relevant in compact mirror based systems. At the same time physical optics, although computationally intensive, is also a useful tool when detailed analysis is required, particularly for providing crosspolarisation information and high accuracy far-field beam patterns from large reflecting antennas, for example. However, modal analysis in general is a very powerful tool, which enables one also to understand issues associated with throughput when partially coherent systems are being considered. This is important for the efficient optical modelling of large arrays systems now being developed for next generation astronomical instrumentation. In the presentation, we will discuss these issues and present examples from real instrumentation. We also summarise our continuing work on the development of computationally efficient modelling tools for fast quasi-optical design and analysis.
\end{abstract}

Keywords: Terahertz, Quasi-Optical Modelling, Gaussian Beam Modes

\section{INTRODUCTION}

Optical design and analysis in the THz waveband can be challenging and difficult to do with any confidence using techniques developed for visible wavelengths, especially for high-precision applications. Generally we wish to calculate an electromagnetic field over some surface in an optical system when the field, or currents, over another surface are known. The source field and the full solution to Maxwell's equations can be extremely difficult to find and in practice approximations have to be made. Physical Optics (PO) refers to the calculation of the field radiated by a reflector using an approximate surface current distribution determined from the incident magnetic field. Central to this method is the assumption that the field on that part of the reflector not directly illuminated by the incoming field is zero. PO has been implemented successfully in the industry-standard commercial package GRASP ${ }^{1}$. Although PO can be used to characterise electromagnetic systems to high accuracy, it is computationally intensive at $\mathrm{THz}$ frequencies and often not suitable for the initial design or preliminary analysis of large multi-element optical systems. An alternative is to decompose one component of the source field into modes, each a solution to the paraxial wave equation. Propagation then simply involves recombining scaled modes with an appropriate mode-dependent phase-slippage term included. Commonly-used mode sets include Gaussian Beam modes (GBM) ${ }^{2}$, Gabor modes and plane waves.

*creidhe.osullivan@nuim.ie, Tel. +353-1-7083953

Terahertz Technology and Applications II, edited by Kurt J. Linden, Laurence P. Sadwick, Créidhe M. M. O'Sullivan, Proc. of SPIE Vol. 7215, 72150P · (c) 2009 SPIE · CCC code: 0277-786X/09/\$18 · doi: 10.1117/12.810807 
In the next section we give a review of GBM theory. We follow this with a very brief description of the THz modeling software that we are developing, software that makes extensive use of beam mode techniques. We use the GBM features for calculations where speed and reasonable accuracy are required, especially for optimisation in the design stages of projects and, for example, tolerance analysis once the design is fixed. In Section 4 we describe the results of some beam optimisation simulations that involve the use of the Gaussian beam mode technique. Finally we introduce some recent work on modal analysis, in particular as it applies to the efficient modeling of telescopes and imaging arrays.

\section{GAUSSIAN BEAM MODES}

Gaussian beam modes constitute complete orthonomal sets that are each solutions to the paraxial wave equation. Any arbitrary solution of this wave equation can therefore be expressed as a superposition of Gaussian modes, the particular mode set, $\Psi_{i}$, being chosen as appropriate for the symmetry of the problem (e.g. Gauss-Hermite or Gauss-Laguerre functions);

$$
E(\boldsymbol{r})=E(x, y, z)=\sum_{i} A_{i} \Psi_{i}(x, y, z ; W(z), R(z)) e^{j\left(\Delta \phi_{i}(z)\right)}
$$

where $E(\boldsymbol{r})$ is the paraxial field, $A_{i}$ are the mode coefficients, $W(z)$ is the beam radius parameter, $R(z)$ is the phase radius of curvature and $\Delta \phi_{i}$ is the phase slippage term. The choice of the optimum beam mode set is crucial to the efficiency of the GBM approach. Because of the limited spatial frequency content of many long-wavelength systems, sources can generally be represented to a high accuracy by the sum of only a few modes and so Gaussian beam modes provide an efficient and natural basis set with which to describe propagation. Once the mode coefficients are known, it is straightforward to model the propagation of a beam by simply keeping track of the evolution of the beam radius, the phase radius of curvature and the phase slippage between modes (using, for example, ABCD matrices), and re-summing them at the plane of interest.

If the field is known over the surface $\Sigma$, then the mode coefficients are determined by calculating the overlap integrals

$$
A_{i}=\iint_{\Sigma} E(x, y, z) \Psi_{i}^{*}(x, y, z ; W, R) d \Sigma .
$$

This decomposition of a field can be a computationally intensive step, but it only has to be carried out once if there is no scattering of power between modes (if mirrors and lenses are treated as perfect phase transformers with no truncation). Scattering of power between modes can be described using the usual Gaussian mode scattering matrix $\mathbf{S}$, where the mode coefficients of the scattered beam are given by

$$
\mathbf{B}=\mathbf{S} . \mathbf{A}
$$

and $\mathbf{A}$ and $\mathbf{B}$ are column vectors of mode coefficients. In the case of truncation by an aperture in the plane perpendicular to propagation, the elements of the scattering matrix can be calculated as

$$
S_{i j}=\iint_{\Sigma} \Psi_{i}^{*}(x, y, z ; W, R) \Psi_{j}(x, y, z ; W, R) d \Sigma .
$$

However if an optical element introduces a significant amount of power scattering between modes, then the number of integrations needed to derive a scattering matrix in a straightforward way can be prohibitive. If an off-axis mirror is treated as an inclined phase-transforming plane then it is necessary to determine the mode coefficients of the scattered field over a plane that is not orthogonal to the direction of propagation and therefore over which the mode set is not orthogonal $^{3}$. An alternative to calculating overlap integrals is to attempt to fit a linear combination (coefficients $\widetilde{\boldsymbol{A}}$ ) of the mode-set functions $\Psi_{i}$ to the known field $\boldsymbol{E}$ at a limited number of sampled points $\boldsymbol{r}_{j}(j=1 \ldots M)$ :

$$
\widetilde{\boldsymbol{E}}=\boldsymbol{\Psi} \tilde{\boldsymbol{A}}
$$

If we minimise the residual of the solution $|\boldsymbol{E}-\widetilde{\boldsymbol{E}}|$, the coefficients $\widetilde{\boldsymbol{A}}_{\boldsymbol{i}}$ approximate the accurate mode expansion coefficients $\widetilde{\boldsymbol{A}}$. The problem can be reformulated using the pseudo-inverse (Moore-Penrose generalized inverse) $\Psi^{+}$of the mode matrix $\Psi$ as

$$
\widetilde{\boldsymbol{A}}=\Psi^{+} \boldsymbol{E}
$$


This solution can be shown to be the best approximation in the least squares sense as well as having the minimum vector norm. Singular value decomposition, or SVD, is one of the most powerful and efficient set of techniques for finding the pseudo-inverse, and we have chosen to use it in our optical modelling software $\operatorname{MODAL}^{4}$ ( $\$ 3$ and see e.g. Press ${ }^{5}$ for details on implementing the method). The SVD approach is generally numerically stable and can deal with both overand under-determined problems.

The propagation of partially coherent fields using GBMs has also been described ${ }^{6}$. We assume that the field under investigation is one member of an ensemble of fields constructed from a set of coherent diffracting free-space modes and, as with a coherent field, it can be expanded in terms of a sum of modes

$$
E^{i}(\boldsymbol{r}, \omega)=\sum_{m} A_{m}^{i}(\omega) \psi_{m}(\boldsymbol{r}, \omega),
$$

so long as the bandwidth is sufficiently small that the phase at one point in a member of the ensemble is well-defined in respect to the phase at any other point in the same field. The cross spectral density $W$ is then

$$
W\left(\boldsymbol{r}^{\prime}, \boldsymbol{r}\right)=\left\langle E^{i}(\boldsymbol{r}) E^{i^{*}}\left(\boldsymbol{r}^{\prime}\right)\right\rangle=\sum_{m} \sum_{n} C_{m n} \Psi_{n}^{*}\left(r^{\prime}\right) \Psi(r)_{m}
$$

where $C_{m n}=\left\langle A_{n}^{i^{*}} A_{m}^{i}\right\rangle$ are the elements of the coherence matrix. The coherence matrix characterises the form of a field at any plane, with all the second-order statistical properties being completely specified. In terms of the cross spectral density, the elements of the coherence matrix are given by

$$
C_{m n}=\int_{S} W\left(\boldsymbol{r}^{\prime}, \boldsymbol{r}\right) \Psi_{n}\left(\boldsymbol{r}^{\prime}\right) \Psi_{m}(\boldsymbol{r}) d s d s^{\prime}
$$

where the expansion functions $\Psi_{\mathrm{m}}$ form an orthonormal basis set and $s$ is the source. For an incoherent field of uniform intensity

$$
\begin{aligned}
& W\left(\boldsymbol{r}^{\prime}, \boldsymbol{r}\right)=I_{o} \delta\left(\boldsymbol{r}^{\prime}-\boldsymbol{r}\right) \text { and } \\
& \boldsymbol{C}=I_{o} \boldsymbol{I} ;
\end{aligned}
$$

all the modes are excited equally and independently. For a completely coherent field the coherence matrix can be simply calculated from the ordinary mode coefficients as

$$
\boldsymbol{C}=\boldsymbol{A} \boldsymbol{A}^{* T} \text {. }
$$

A partially coherent field can be traced through a submillimetre-wave optical system using the overall coherent-mode scattering matrix $\boldsymbol{S}$ of the system. Withington $\&$ Murphy ${ }^{6}$ have shown that

$$
\boldsymbol{D}=\boldsymbol{S C S} \boldsymbol{S}^{* T}
$$

where $\boldsymbol{C}$ is the coherence matrix at the input plane of the system and $\boldsymbol{D}$ is the coherence matrix at the output plane. Just as in the coherent case where the fields are decomposed into modes and then propagated from one plane to another, in the more general case the cross spectral density is broken down into modes and it is these modes (or elements of the coherence matrix) that are propagated. The intensity distribution of the field can then be recovered from the coherence matrix.

\section{MODAL}

In general there is a lack of dedicated software tools for modelling the range of components and propagation conditions encountered in typical quasi-optical $\mathrm{THz}$ systems and we have had to employ a variety of commercial and in-house software packages for this task. We are developing our own software analysis package: 'MODAL' (Maynooth Optics Design and Analysis Laboratory ${ }^{4}$ ) (Fig. 1) which employs approximate (GBM) methods for visualisation and optimisation during the design stages, and a more rigorous vector PO technique for the final analysis. MODAL provides a range of optical elements; those implemented so far include idealised sources, corrugated conical horns, mirrors, 
apertures, dielectric lenses and phase gratings. The available simulation methods are scalar diffraction integrals, vector PO and modal propagation using Gaussian-Hermite beam modes (and the SVD technique described in $\S 2$ ). The range of elements implemented to date has allowed us to accurately model Cosmic Mocrowave Background (Fig. 1) and other telescopes with MODAL. Of particular interest to us are the polarisation properties of these telescopes and the understanding of any elements that are likely to introduce instrumental or cross-polarisation.

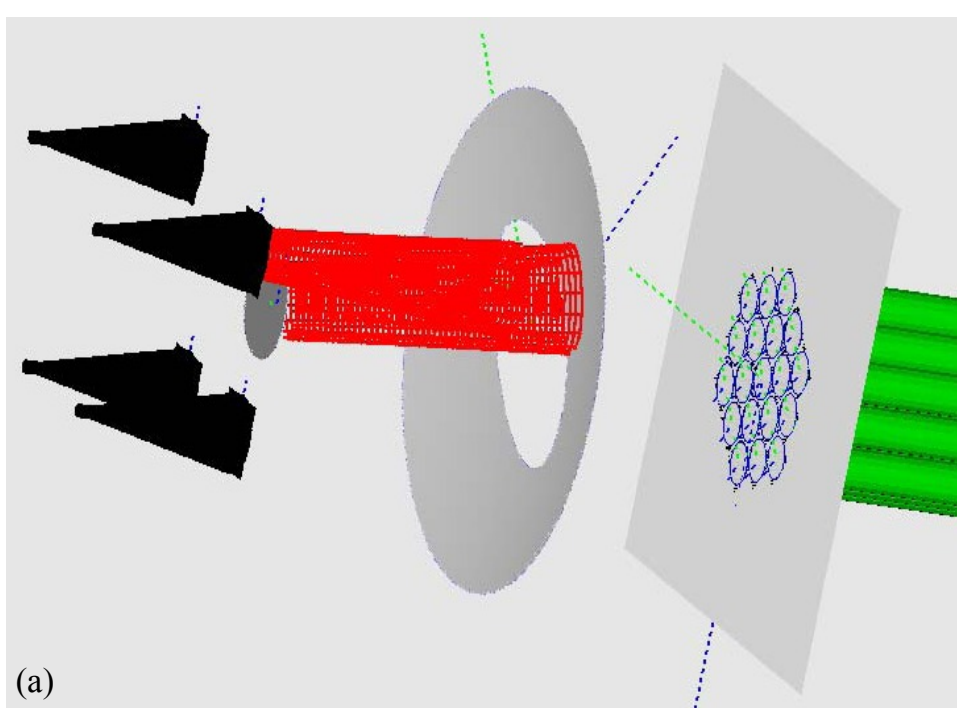

Fig. 1. (a) MODAL model of the optical beam combiner in a bolometric interferometer $(\mathrm{MBI})^{7}$. GBMs are used for preliminary design and analysis. A fundamental Gaussian beam can be used for visualisation. The output (in this case a fringe pattern at the focal plane) can be calculated using (b) full vector physical optics and (c) GBMs.

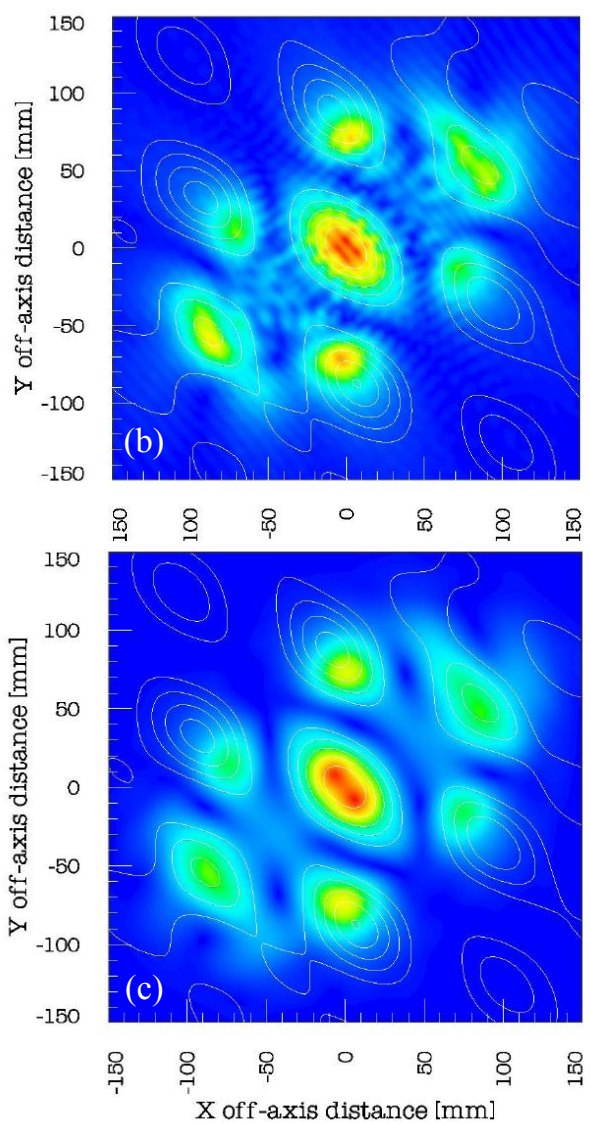

\section{BEAM CONTROL AND OPTIMISATION}

Diffractive Optical Elements (DOEs) which, for example, transform a field distribution at a focal-plane to a prescribed intensity distribution in the far-field, can be readily designed using $\mathrm{GBMs}^{8}$. (Here we assume that the light incident on the DOE is coherent and monochromatic.) DOEs impose a phase on the field (using a dielectric material, or a profiled reflector), thereby creating the desired interference pattern at some distance from the DOE. The design of DOEs is essentially the same problem as phase retrieval, and the same algorithms that are used to design DOEs can be used to recover the phase of a field from intensity-only measurements at two planes ${ }^{9}$. For beam shaping, the problem is to determine the phase distribution which, when imposed on the input field, produces the required far-field distribution. Although the same algorithms are used, there are some differences in the nature of the two problems. With phase retrieval one is generally interested in retrieving the phase from noisy data, and a solution is known to exist. However, with DOE design an exact solution may not exist ${ }^{10}$ and if it does it may not be unique or stable ${ }^{11}$.

A common method used in DOE design is the iterative approach of the Gerchberg Saxton Algorithm (GSA $\left.{ }^{11,9}\right)$. The algorithm propagates a field between the object and Fraunhofer planes (using the FFT), replacing the amplitude with the target amplitude at each plane, while retaining the phase. The intensity distribution of the solution has been shown to become closer to the target with each iteration ${ }^{12}$. Fig. 2 shows an example where we have used the GSA to find the 
phase (Fig. 2 (a)) that must be imposed at the waist of a Gaussian beam to transform it into a super-Gaussian beam (Fig. 2 (b)), of order 20, in the far-field.
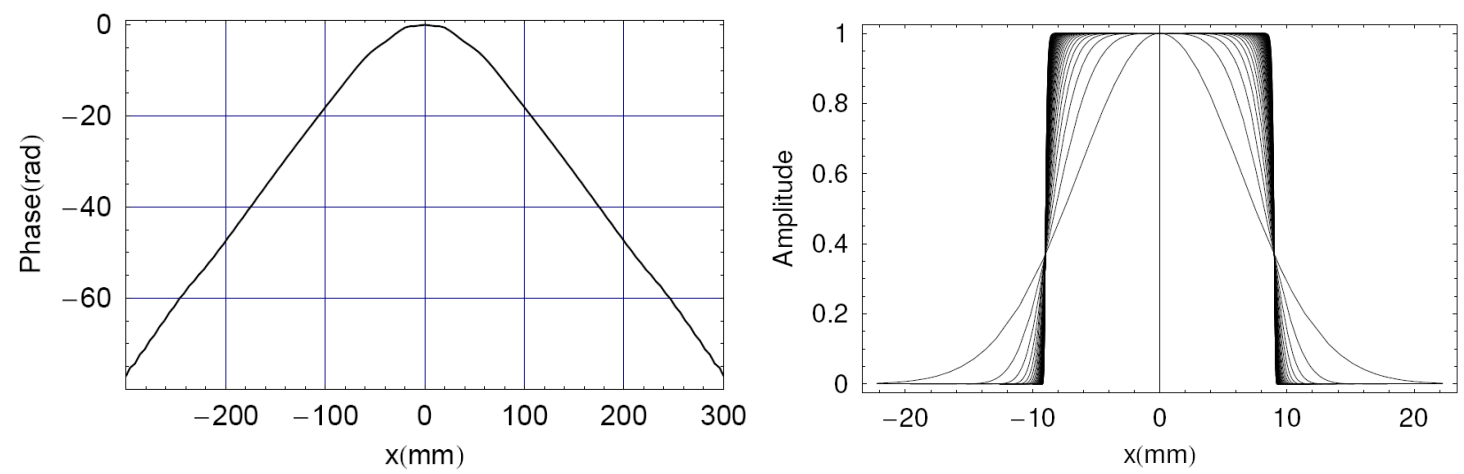

Fig. 2. (a) The near-field phase (giving the DOE surface profile) corresponding to the solution with the lowest mean squared error for a Gaussian to 20th order super-Gaussian transformation. (b) Plot of super-Gaussian functions, $\exp \left(-\left(\frac{x}{w}\right)^{n}\right)$, of order $\mathrm{n}=2,4, \ldots 98,100 . w$ was taken as 9 .
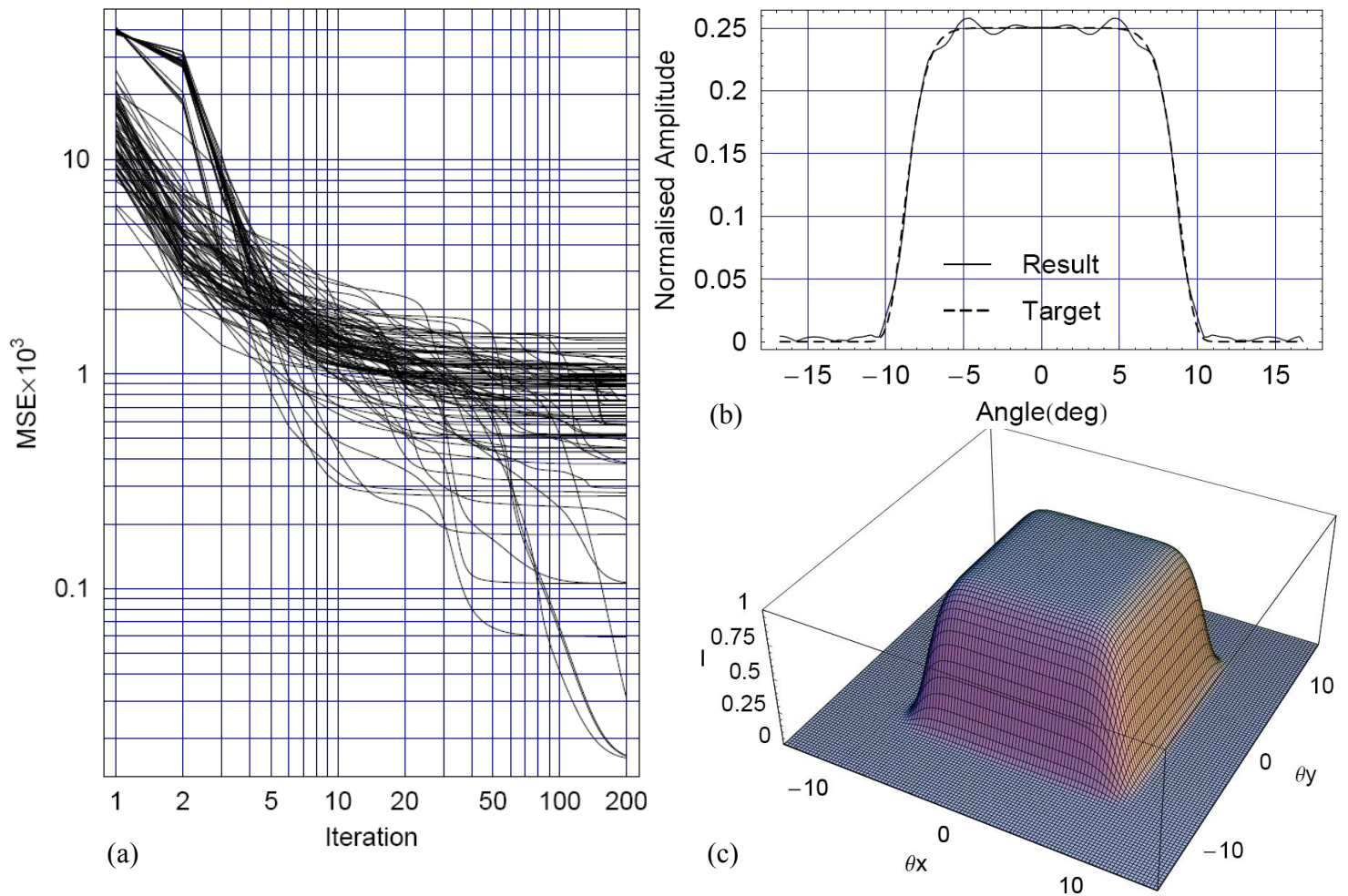

Fig. 3. (a) The progress of 100 trials of the GSA, for a DOE that converts a Gaussian to a super-Gaussian of order 20. (b) The far-field amplitude distribution (1d and (c) 2d) corresponding to the solution with the lowest MSE for a Gaussian to $n=20(w=9)$ super-Gaussian transformation.

Fig. 3(a) shows the progress (in terms of the mean-squared error) of the GSA for 100 trials with random initial phase distributions. As with other techniques, success depends on the function being minimised, the sampling and the initial estimate. A large number of initial trials tend to converge to a significantly smaller number of solutions however. In this 
example we ran 100 trials. The output amplitude distribution that can be obtained with a DOE surface profile developed from Fig. 2(a) is shown in Fig. 3(b) \& (c).

In an earlier paper ${ }^{13}$ we applied this technique to the design of Fourier gratings using the FFT algorithm to propagate the input beam to the far-field. We found good solutions using the Gerchberg-Saxton/FFT algorithm but in most cases the grating pattern consisted of complicated repeat patterns or patterns with a lot of fine detail. The success of the phase retrieval algorithms in these cases relied on the large number of degrees of freedom provided by the FFT. However, for simple output patterns, the FFT has perhaps too many degrees of freedom, resulting in highly complicated gratings that would be difficult to manufacture. Many of the grating features in these solutions are in fact smaller than the illuminating wavelength and probably do not contribute much to the desired output pattern. An alternative is to replace the method of field propagation between the object and image plane (the FFT), with one using Gaussian beam modes. Limiting the number of modes automatically reduces the complexity of the solutions allowed and yet can provide satisfactory far-field beam patterns. It has the advantage over Fourier techniques that the plane where the phase grating is located does not have to be exactly the Fourier plane.

An alternative GBM technique is described by Isaak et al. ${ }^{14}$ in their paper on phase retrieval. They described the object and target fields in terms of Gaussian beam modes and minimised, using the Levenberg-Marquardt algorithm, the meansqaure error between the target and trial intensity distributions on the two planes by adjusting the complex mode coefficients. The technique was extended to partially coherent fields ${ }^{15}$. We found that their optimisation techniques worked well for phase retrieval but not so well for beam shaping (where exact solutions may not exist). We have used the same general method, varying the parameters which encode the phase imposed on the input field in order to find the solution which results in the best match between the target and predicted distributions, but have investigated global optimisation algorithms such as differential evolution $(\mathrm{DE})^{16}$ and simulated annealing (SA) ${ }^{17}$ SA operates on a single set of parameters to be optimised, DE uses a population of parameters. Fig. 4 compares the results for a DOE designed to convert a single input Gaussian beam to a $3 \times 3$ array of Gaussian beams in the far field.

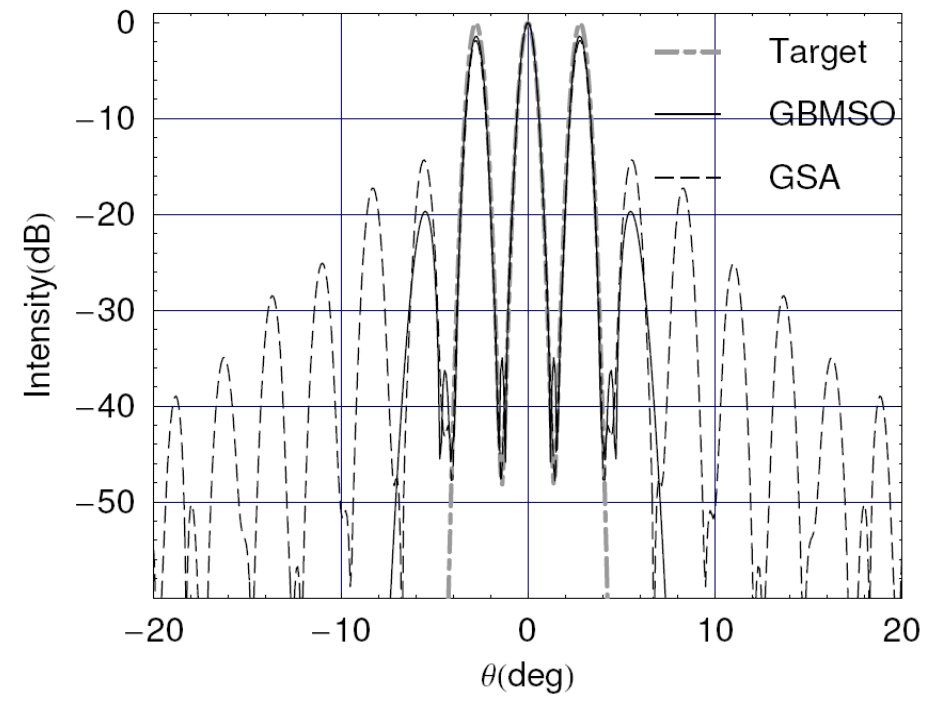

Fig. 4. Simulated far field (1d cut) of a phase grating designed to convert a single Gaussian beam to a $3 \times 3$ array of beams. Gratings were designed using the best of 100 trials of the Gerchberg-Saxton Algorithm (GSA) and by Gaussian Beam mode set optimisation (GMBSO).

Another significant advantage of the Gaussian beam mode methods is that they are not restricted to two planes and so can be applied to problems where we want to control beams in the near-field or along their propagation paths. As an example we have investigated the production of non-diffracting beams. So-called "diffraction-free beams", in which the amplitude does not change in form or scale while propagating, were identified in $1987^{18}$. They have the amplitude crosssection of a Bessel function

$$
E(r, z)=E_{o} \exp \left(i k_{z} z\right) J_{0}\left(k_{r} r\right)
$$


where $J_{0}$ is the zeroth-order Bessel function, $k_{z}$, and $k_{r}$ are the longitudinal and radial components of the free-space wave vector, and $k_{z}^{2}+k_{r}^{2}=k_{0}^{2}$, where $k_{0}$ is the free space wavenumber. Bessel beams have been used, for example, to provide a very large depth-of-field in imaging systems and radiation-pressure particle traps ${ }^{19}$. Ideal Bessel beams have infinite extent and power; real "pseudo-Bessel beams", having finite extent and power, can only approximate them. PseudoBessel beams have been generated using dielectric conical shaped lenses called axicons ${ }^{20,21}$ in the region of overlap of two beams it produces (Fig. 5). Beyond this region the beam diffracts rapidly.

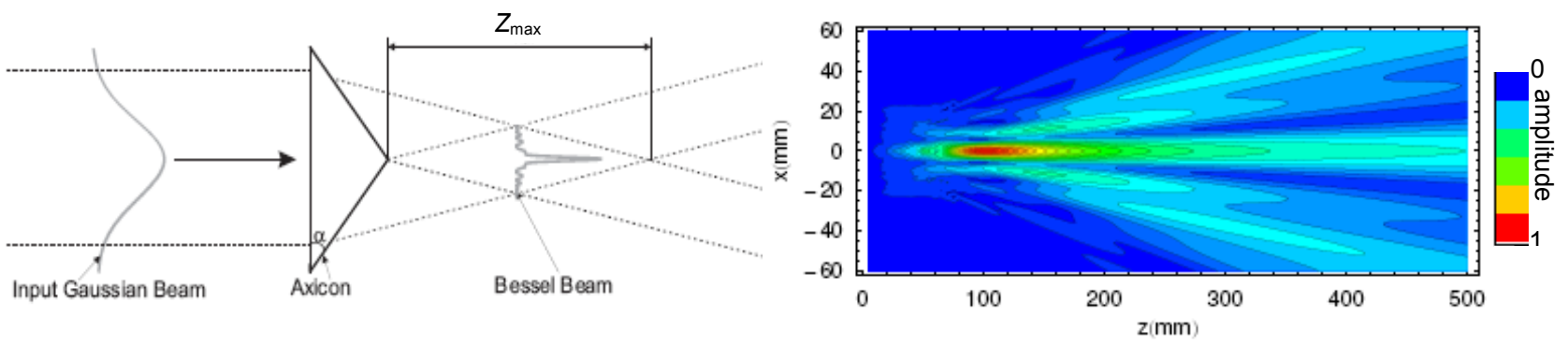

Fig. 5. (a)An input beam is transformed into a pseudo-Bessel beam within the region $Z_{\max }$.(b) Simulation of the field produced by an axicon. (This simulation agrees closely with the field from a HDPE axicon with slant angle $\alpha=$ $20^{\circ}$ and a radius of $30 \mathrm{~mm}$. It was illuminated with a $78 \mathrm{~mm}$-radius Gaussian beam of wavelength $\lambda=3 \mathrm{~mm}$ with a flat phase-front.)

We used a Gaussian beam mode-set optimisation to design other DOEs that create non-diffracting beams $(\lambda=3 \mathrm{~mm}$ in our example). A set of mode coefficients describing the phase imposed on an input Gaussian beam was varied to

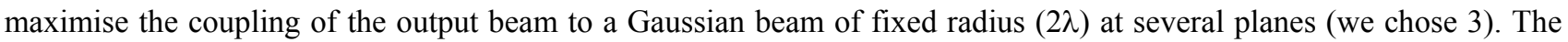
coupling between 2 fields with Gaussian beam mode coefficients $A_{i}$ and $B_{i}$ is simply calculated as

$$
\text { field coupling }=\sum_{i} A_{i}^{*} B_{i}
$$

The waist of the basis mode set was chosen to be large enough so that the mode set did not diffract significantly between the planes of interest. The result is shown in Fig. 6(a). As the target planes, i.e. the planes across which the coupling is calculated, were separated, the field between them became less focused (Fig. 6(b)) and so the merit function was changed to look for constant on-axis power at more than 3 planes (Fig. 6(c)). The same technique can be used to design beams that avoid obstacles in their path (Fig. 7$)^{8}$.
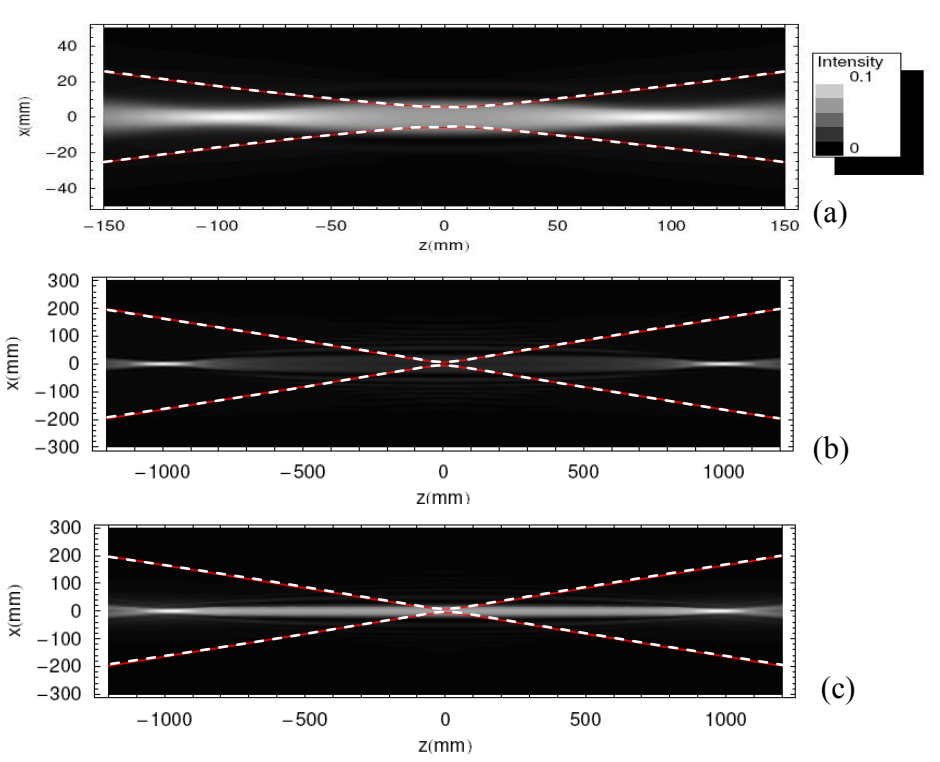

(c)
Fig. 6. (a) Intensity of a propagating "diffraction-free" beam. The phase described by a Gaussian beam mode set was varied so as to give a Gaussian beam of radius $2 \lambda(6 \mathrm{~mm})$ at $\mathrm{z}=0, \pm 100 \mathrm{~mm}$. The dashed lines show the radius of a propagating Gaussian beam with the same radius at $\mathrm{z}=0 \mathrm{~mm}$. (b) As (a) except the target planes were at $\mathrm{z}=0, \pm 1000 \mathrm{~mm}$. (c) As (b) except the mode-set was varied to also try and maintain constant onaxis power. 

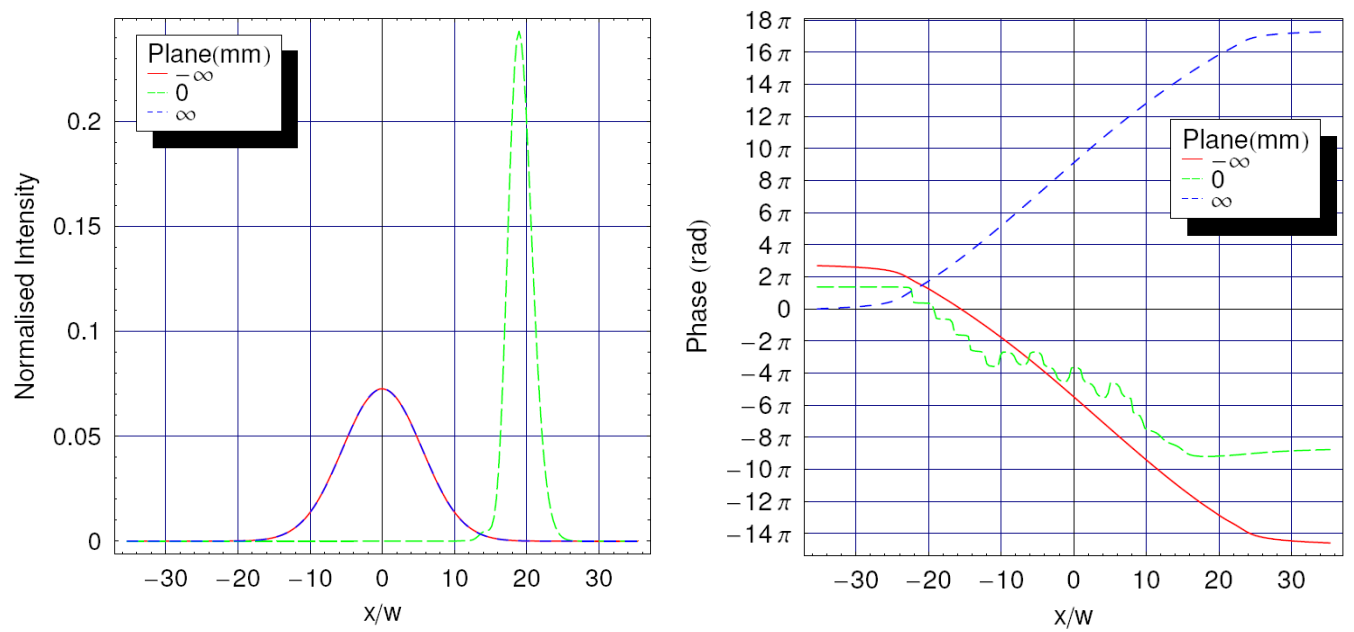

Fig. 7. Intensity and phase of a beam which has a Gaussian amplitude distribution at $z= \pm \infty$, and has no power in the region $x<10 \mathrm{~mm}$ at $z=0 \mathrm{~mm}$. A DOE can be designed from the phase required at any plane.

We found in many cases, like the ones illustrated above, that imposing a phase on an input beam at one plane resulted in an output beam close to the one desired (we have not included the effects of truncation). However, in most cases an exact solution could not be found without modifying both the amplitude and phase of the input beam. An alternative to absorbing power in order to modify the amplitude distribution is to use two phase-transforming surfaces or DOEs, one to produce a suitable amplitude distribution at a second plane where a further phase distribution is imposed (Fig. 8). Fig. 9 compares the results that can be obtained using one or two surfaces for a DOE designed to produce a grid of beams from one input beam.
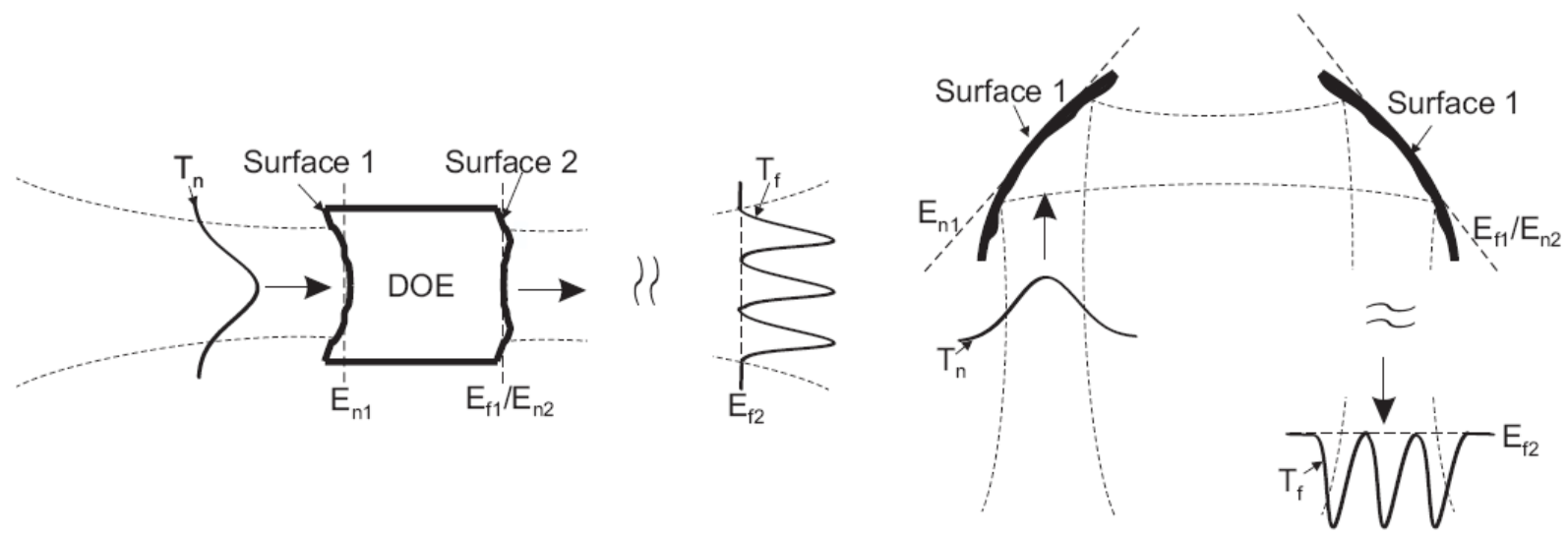

Fig. 8. Beam shaping using two surfaces (a) in transmission, using a dielectric material and (b) in reflection, using shaped mirrors. The figure shows an example of a DOE that converts an incident Gaussian beam to three beams in the far-field.

We have used this technique to see if the coupling of the field from the sky to the detector horn could be improved in Band $9(\sim 660 \pm 60 \mathrm{GHz})$ of the ALMA telescope. The Atacama Large Millimeter Array (ALMA) interferometer is a 50element array of 12-m Cassegrain telescopes being built in Chile by an international collaboration involving the European Southern Observatory, the Japanese National Institutes of Natural Sciences and the National Radio Astronomical Observatory in the US ${ }^{22}$. The optics in Band 9, designed using ray-tracing techniques, allow frequency independent coupling between the telescope and mixer horn. The two elliptical mirrors M3 and M4 (Fig. 10(a)) re-image the mixer horn aperture to the secondary mirror. 

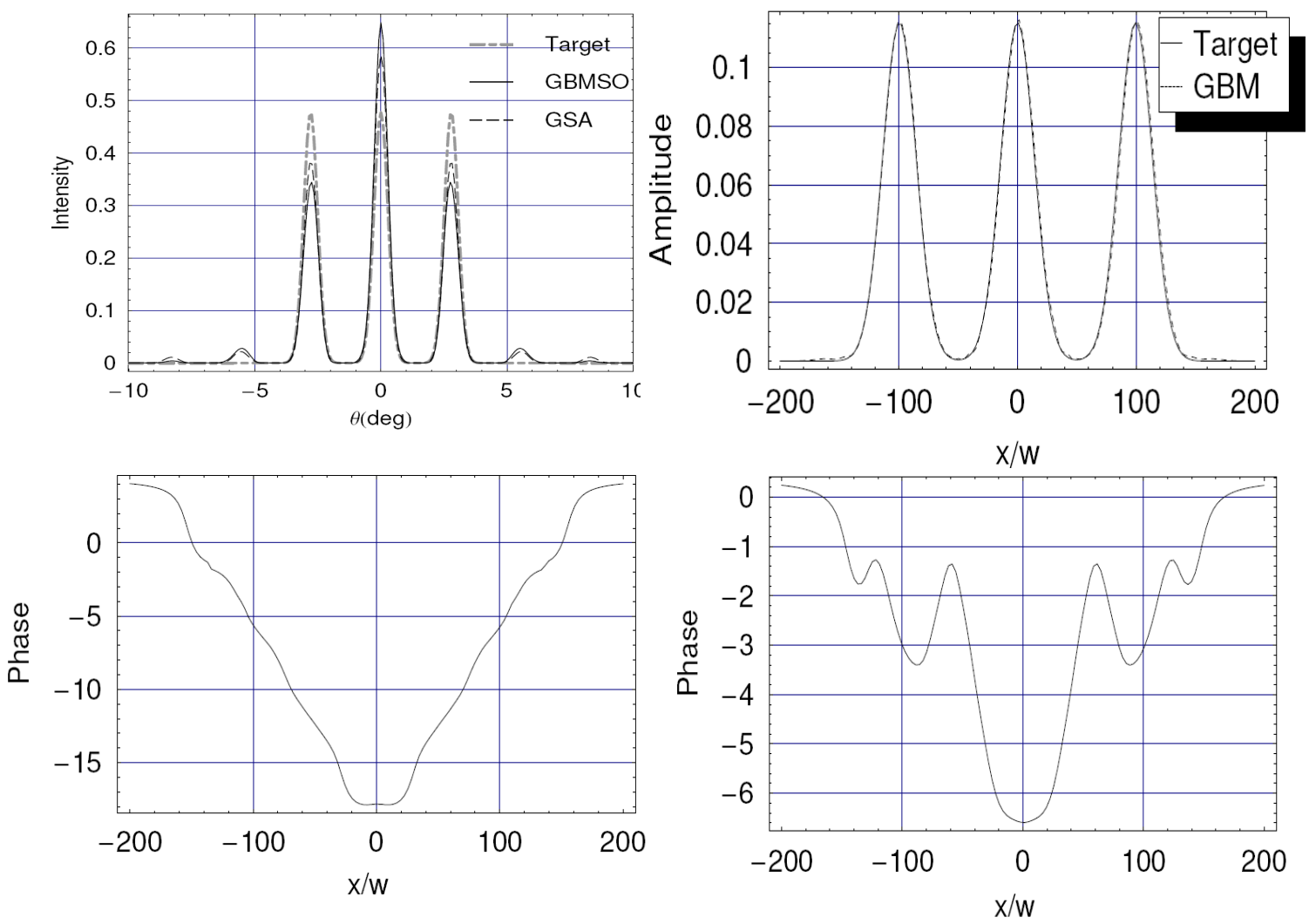

Fig. 9. Simulated far field (1d cut) of a DOE designed to convert a single Gaussian beam to a $3 \times 3$ array of beams using (a) one phase-transforming surface (the example of Fig. 4) and (b) two surfaces. (The scale of the output beams was left as a variable in this optimisation.) (c) and (d) show the phase, in radians, that must be imposed on the beam at the 2 intermediate planes chosen.

A dielectric phase plate, which produces a phase delay proportional to its optical depth, can be designed to improve the coupling between the field from the sky and the mixer horn. (Alternatively the profile of the phase plate can be machined into the surface of mirror M3, for example.) The field from the secondary mirror can be approximated by a top-hat distribution with an aperture radius of $375 \mathrm{~mm}$ and a phase radius of curvature of $6 \mathrm{~m}$. The ALMA optics were designed to match as closely as possible the phase-front of the field from the secondary mirror to the phase-front of the mixer horn-field. However, as shown in Fig. 10 (b), the fields cannot be matched exactly. If only one surface is used to impose a phase then the maximum coupling that can be achieved is when the phases of the two fields are matched. The surface profile needed to do this was calculated using the Gaussian Beam mode optimisation method described previously. This profile, and the coupling that can be achieved, varies depending on the plane where the phase-matching is done (Fig. 11). Currently, about $88 \%$ of the radiation from the sky is coupled to the mixer horn. Profiling the mirror M3 could increase this to $93 \%$ (close to the maximum). Profiling a mirror has the additional benefit of not introducing any new optical components into the system. By using both mirrors M3 and M4, both the amplitude and phase of the field from the horn can be controlled to achieve a power coupling of $97.8 \%$ (this analysis was carried out in one-dimension for simplicity). This compares with the maximum possible power coupling of $98.4 \%$; $100 \%$ coupling cannot be achieved due to the truncation of the horn-field at M3. Complete control of the field can be achieved with three mirrors: two beam shaping mirrors and one phase matching mirror; allowing a field with any specified amplitude and phase distribution to be produced at the given plane. However, while diffractive elements can be designed to couple the all radiation at a particular frequency, the shape of the mirrors is not ideal at other frequencies, restricting their usable bandwidth. We have found optimisation using GBMs to be a useful approach as it allows the beams to be defined using a small number of parameters, and small numbers of GBMs result in designs with smooth profiles. 

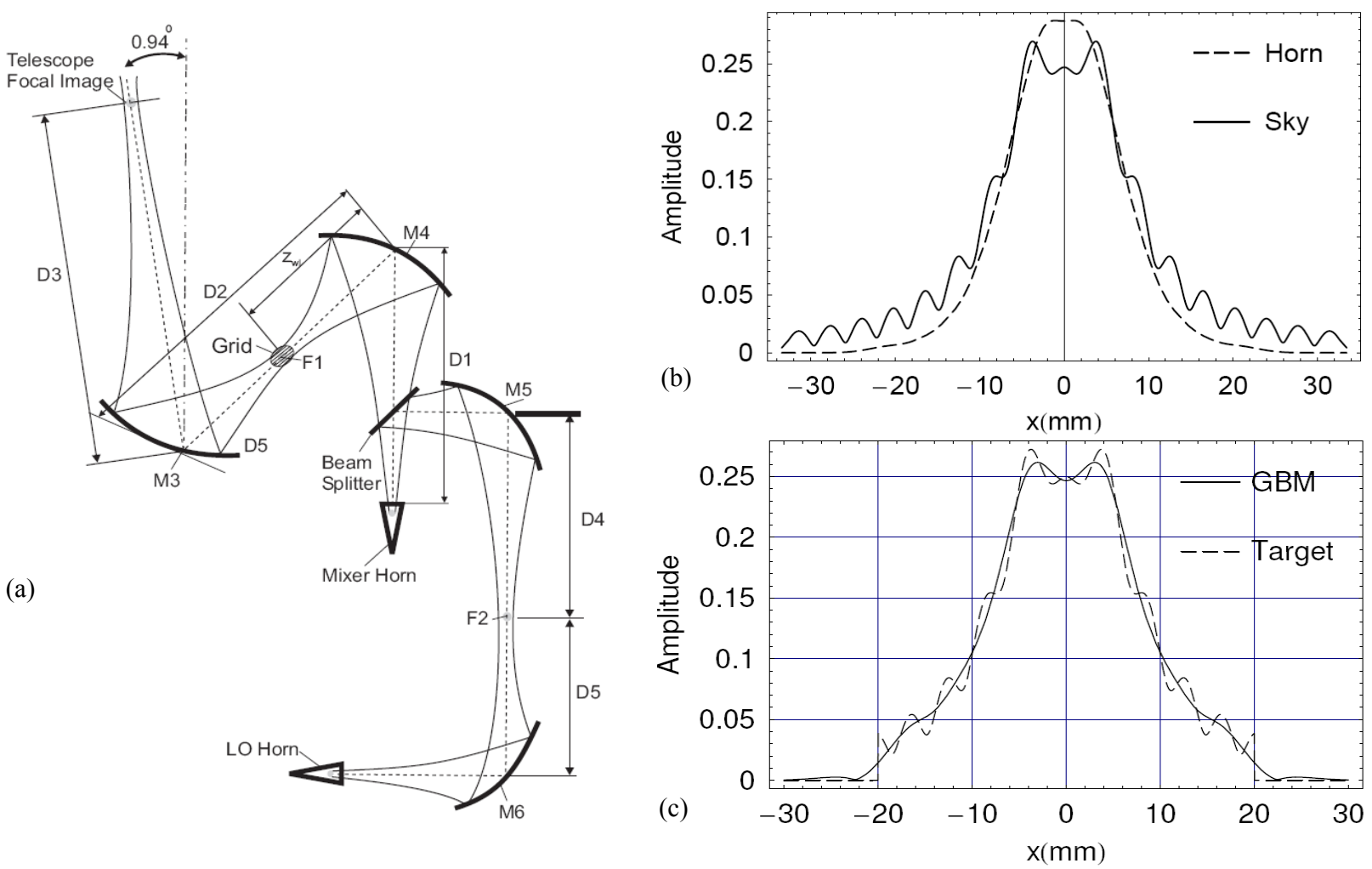

Fig. 10. (a) ALMA Band 9 optical configuration. (b) The tield trom the mixer horn and from the sky, both propagated to mirror M3 (diameter $40 \mathrm{~mm}$ ) using the current optical configuration. (c) The fields at mirror M3 after phase-matching at two-surfaces.

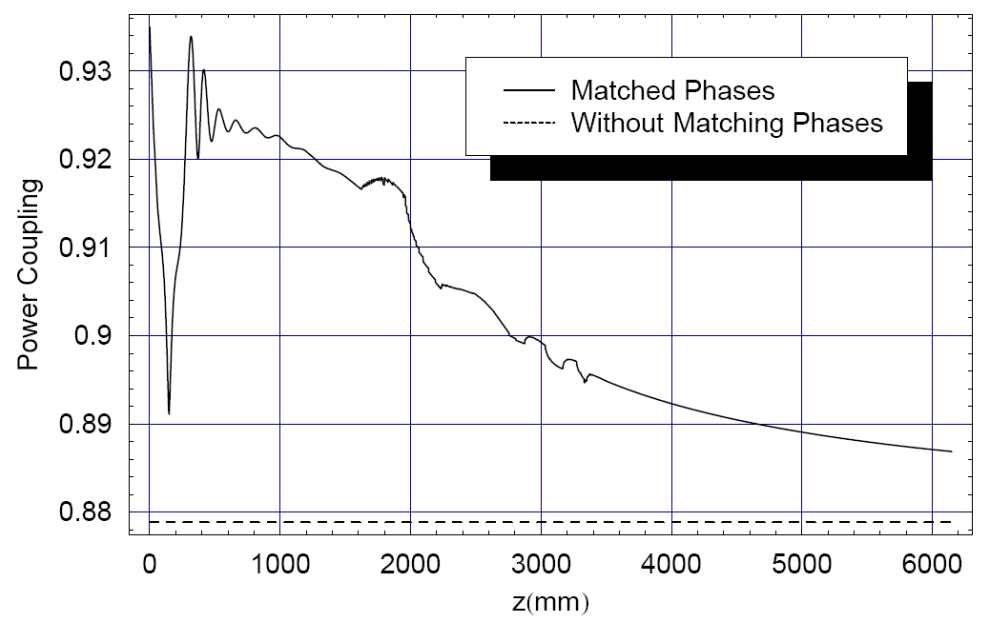

Fig. 11. The solid line shows the achievable power coupling as a function of phase plate distance from M3, at $0 \mathrm{~mm}$. The secondary mirror is $6149.44 \mathrm{~mm}$ from M3. The phase plate is designed to match the phases of the two fields. The dashed line shows the coupling between the fields when the phases are not matched.

\section{BASIS MODE SETS}

A modal analysis can be extremely useful when designing efficient quasi-optical systems or when trying to verify them in an efficient way. In the case of the GBM analysis, it is possible to select a finite, often small, set of modes that is a nearly complete basis set for the propagating beam. If mode coefficients are to be calculated by fitting a linear 
combination of modes to a known field, then only a relatively small number of sample points may be needed. Problems can arise, however, due to the non-orthogonality of modes (for modes over a curved surface, or inadequately sampled modes, say). Curran et al. ${ }^{7}$ describe how the robustness of the approach can be improved by using a truncated SVD decomposition of the matrix that is constructed from the potential mode set, sampled at a discrete number of points. SVD decomposes an $m \times n$ matrix $\mathbf{S}$ into the product of an $m \times n$ column-orthogonal matrix $\mathbf{U}$, an $n \times n$ diagonal matrix $\mathbf{W}$ (whose elements $w_{i}$ are $\geq 0$ and termed the singular values) and the transpose of an $n \times n$ orthogonal matrix $\mathbf{V} ; \mathbf{S}=\mathbf{U W V} \mathbf{V}^{\mathrm{T}}$. SVD analysis allows the effective rank of the mode matrix to be determined and, by looking at the singular values, problematic modes can be removed from the decomposition. ${ }^{23}$

The truncation in many optical systems is not severe and the mode set used to propagate beams in free space is a good choice for the basis set describing the beam throughout the system. ${ }^{24}$ However cases do arise, for example if a waveguide structure is embedded in a free space quasi-optical system, where the number of independent modes that can propagate through the system is much smaller than the number of modes that need to be considered at its input. ${ }^{7}$ The most efficient way of modelling the system is to determine the combinations of input modes that give rise to non-zero output mode combinations, as only these few combinations then need to be coupled with the incident field. Singularvalue decomposition of the waveguide scattering matrix can be used to find those effective modes at its input and output ports, as well as their attenuation as they propagate through the structure. The columns of $\mathbf{U}$ corresponding to non-zero singular values are an orthonormal set of basis vectors that span the range of output modes, while the same columns of $\mathbf{V}$ are an orthonormal set of basis vectors that span the range of input modes In the analysis of a back-to-back waveguide structure by Curran et al., ${ }^{22}$ only a small number of singular values are effectively non-zero and the corresponding columns of the $\mathbf{V}$ matrix give the only independent combinations of the input waveguide modes that need to be considered. Similarly, Withington et al. ${ }^{25}$ show how the behaviour of bolometric interferometers can be efficiently modeled by determining ${ }^{24}$ the natural modes or eigenfields of the system.

Withington et al. ${ }^{26}$ have developed a general theory for analysing imaging phased arrays that allows overlapping beams to be modeled. Their technique involves the identification of orthogonal field patterns to which the array can couple (i.e. its natural modes), using a singular-valued decomposition of the synthesised beams of the array (similar to $\mathbf{V}$ above). The dynamic range of the system determines the threshold below which the singular values are not considered. Once these modes are identified, dual beams can be constructed (since the synthesized beams may not be orthogonal) and sources recovered from measured correlations.

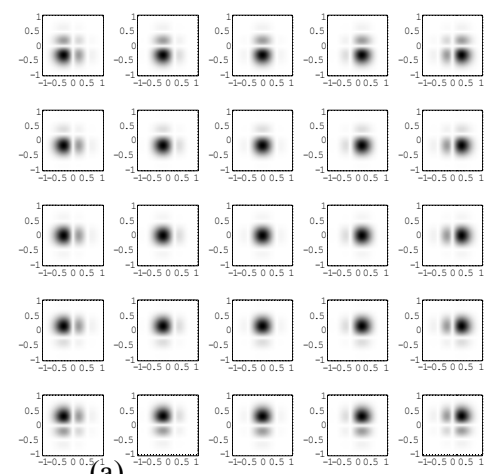

(a)

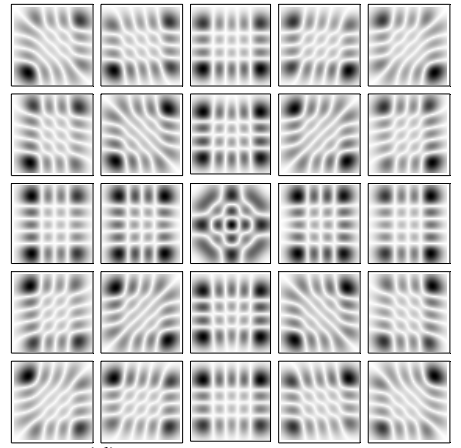

(d)

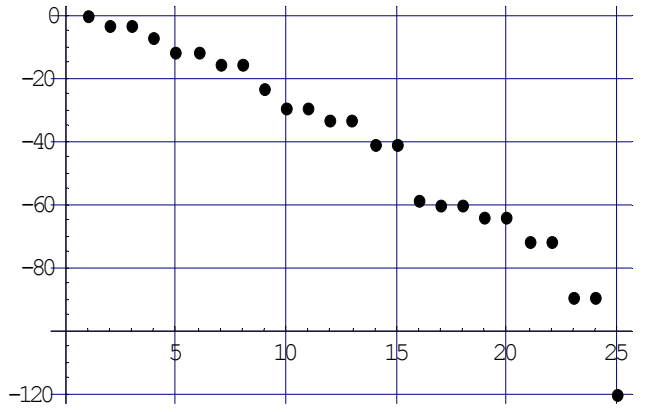

(b)

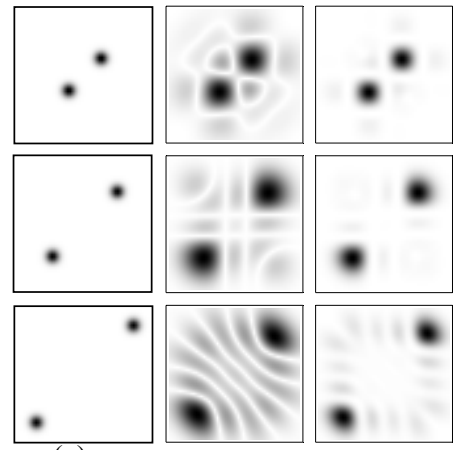

(e)

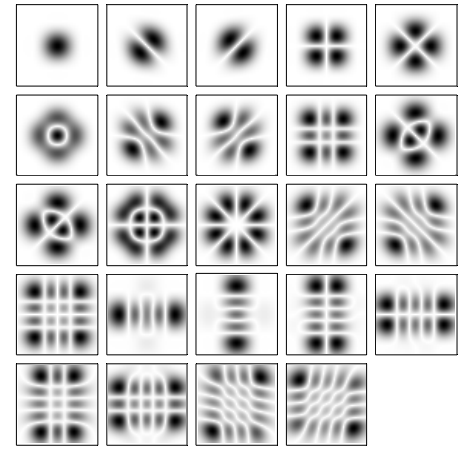

(c)

Fig. 12. Example from Saklatvala at al. ${ }^{27}$ (a) Synthesised beams of a $10 \lambda \times 10 \lambda$ phased array of $5 \times 5$ horns with a Bulter matrix. (b) Singular values from the SVD of the beams in (a). (c) The eigenfields of the array (the field corresponding to the lowest singular value is not included). (d) The dual beams, (e) (middle) the magnitude of a reconstructed coherent source (left); and (right) the intensity of a reconstructed incoherent source. 


\section{CONCLUSION}

Gaussian beam modes are a computationally efficient means of analyzing THz quasioptical systems. Although not as rigorous as a full vector physical optics calculation, they are sufficient for many of the finite-throughput optical systems used in practice and often give additional physical insights into their behaviour. The technique can be extended, using coherence matrices, to model partially coherent fields. We have chosen beam control and optimisation as an example to illustrate their usefulness. Modal analyses in general can help in the understanding and therefore both the design and efficient analysis of complex imaging arrays.

\section{ACKNOWLEDGMENTS}

The authors gratefully acknowledge financial support from Science Foundation Ireland. We thank the members of the HIFI, PLANCK, MBI and QUaD collaborations for continuing helpful discussions.

\section{REFERENCES}

[1] GRASP8, TICRA Engineering Consultants, http://www.ticra.dk

[2] Goldsmith, P.F., "Quasioptical Systems”, IEEE Press, (1998).

[3] Murphy, J.A. \& Withington, S., "Perturbation analysis of Gaussian-beam-mode scattering at off-axis ellipsoidal mirrors", Infrared Physics \& Technology, 37, 205-219, (1996).

[4] Gradziel, M.L., et al., "Modelling of the optical performance of millimeter-wave instruments in MODAL", Proc. SPIE Vol. 6472, THz and GHz Electronics and Photonics VI, K. J. Linden \& L.P. Sadwick eds., 64720D, (2007).

[5] Press, W.H., et al., "Numerical Recipes in C", Cambridge University Press, (1992).

[6] Withington, S. \& Murphy, J.A., "Modal Analysis of Partially Coherent Submullimetre-wave Quasioptical Systems", IEEE Trans. Antennas \& Propagation., Vol. 46, pp. 1650-1658, (1998).

[7] Curran, G.S. et al., "Modeling the quasi-optical performance of CMB astronomical interferometers", Proc. SPIE Vol. 7020, Millimeter and Submillimeter Detectors and Instrumentation for Astronomy IV, 70202O, (2008).

[8] Lavelle, J., "The Design and Optimisation of Quasioptical Telescopes", PhD Thesis, NUI, Maynooth, (2008).

[9] Fienup, J., "Phase retrieval algorithms: a comparison", Applied Optics, 21:2758-2769 (1982).

[10] Soifer, V., et al., " Methods for Computer Design of Diffractive Optical Elements”, Wiley, (2002).

[11] Kim, H. et al. "Iterative Fourier Transform algorithm with regularization for the optimal design of diffractive optical elements", Optical Society of America, 21:2353-2365, (2004).

[12] Gerchberg, R. et al. "A practical algorithm for the determination of phase from image and diffraction plane pictures", Optik, 35:237246, (1972).

[13] O'Sullivan, C., et al., 'Novel applications of Gaussian beam mode analysis', Proc. SPIE Vol. 6120, Terahertz and Gigahertz Electronics and Photonics V, R.J. Hwu \& K. J. Linden eds., 61200J, (2006).

[14] Isaak, K., et al., "Phase retrieval at millimetre and submillimetre wavelengths using a Gaussian-beam formalism", Proc. Fourth International Symposium on Space Terahertz Technology, (1993).

[15] O'Sullivan C., et al., "Coherent and Incoherent Phase Retrieval using Gaussian Beam Modes", Proc. 8th International Conference on THz Electronics, pp. 153-156, (2000).

[16] Price, K., et al., "Differential Evolution", Springer, (2005).

[17] Aarts, E., "Simulated Annealing: Theory and Applications", Springer, (1987).

[18] Durnin, J., "Diffraction-free Bessel beams", Journal of the Optical Society of America, 58:651-654, (1987).

[19] Paterson L. et al." "Light-induced cell separation in a tailored optical landscape" App.Phys.Lett.,87,12:123901 (2005)

[20] Monk, S., el al., "The generation of Bessel beams at millimetre-wave frequencies by use of an axicon", Optics Communications, 170:213-215, (1999).

[21] Trappe, N., et al. "The quasi-optical analysis of Bessel beams in the far infrared", IR Phys. \& Tech., 46:233, (2005).

[22] ALMA website http://www.alma.cl/

[23] Gradziel, M. L. et al., "Fast CAD Software for the Optical Design of Long-Wavelength Systems", IRMMW-THZ, Williamsburg, Virginia, USA, pp. 237-8, (2005).

[24] Murphy J.A. et al., "Optical modeling for millimeter and submillimeter-wave systems", Proc. SPIE, Vol. 7020, Millimeter and Submillimeter Detectors and Instrumentation for Astronomy IV, 70200W, (2008).

[25] Withington S. et al., "A numerical procedure for simulating the behaviour of multimode, bolometric, astronomical interferometers", J. Applied Physics, 97 (12), 124909 (2005).

[26] Withington S. et al., "Theoretical analysis of astronomical phased arrays", J. Opt. A: 10, 015304, (2008).

[27] Saklatvala, G. et al., "Simulations of astronomical imaging phased arrays", JOSA A, Vol. 25, 4, 958, (2008). 